\title{
A suggestion for future research on interface design of an internet-based telemedicine system for the elderly
}

\author{
Young J. Chun ${ }^{\mathrm{a}^{*}}$ and Patrick E. Patterson ${ }^{\mathrm{a}}$ \\ ${ }^{a}$ Department of Industrial Engineering, Texas Tech University, 2500 Broadway, Lubbock, TX 79409, USA
}

\begin{abstract}
Telemedicine has emerged as an effective tool for providing high quality healthcare service and health-related information, especially in rural areas. Rural areas often have a larger elderly population with greater rates of preventable disease. These areas also have fewer medical resources and specialists, and have limited access to health services, all of which can influence overall health. An internet-based telemedicine system can be one solution to provide the rural elderly with the proper health information when needed. The elderly obviously have limited capabilities compared with younger adults in the use of internet technology. However, the interfaces of the currently existing internet-based telemedicine systems are not specifically developed for elderly users. This paper suggests future interface design research for an internet-based telemedicine system specifically for the elderly.
\end{abstract}

Keywords: telemedicine system, internet, interface design, elderly, usability

\section{Introduction}

Telemedicine is becoming a powerful tool for providing high quality healthcare, health-related information, and education in rural areas [23]. Rural areas account for about $20 \%$ of the US population [29], and have fewer resources and specialists, and limited access to health services when compared with urban areas [24]. Rural areas often have large elderly populations, many of whom have preventable disease risk factors such as obesity, smoking, poor diet, lower rate of activity, and consequent higher chronic disease than urban residents $[19,23]$. Further, it is not easy to travel for medical care because of the lack of transportation and the great distances found in rural areas. Telemedicine can be used to provide homebased medical care, education opportunities, or other services to help rural residents get proper and timely information about overall health.

The internet can be an effective tool to deliver health related services [12]. Healthcare services offered over the internet are continuously increasing as an important complement to, and even substitution for, traditional health services [30]. While many population groups may benefit from telemedicine, the elderly are especially likely to do so. That is, an internet based telemedicine system can provide proper health information to the elderly whenever, and wherever, needed.

The elderly population continues to increase dramatically. According to U.S. Census Bureau [29], individuals who are over 55 years old accounted for $22.6 \%$ of the population. The number of elderly over 65 is about 35 million and accounted for $12.1 \%$ of the total U.S. population in 2006. It is expected that the number of older adults will reach 108 million, or $45 \%$ of the adult population, in the next 15 years $[4,13]$.

One reason for the increasing concern for our growing elderly population is that elderly populations suffer a much higher incidence of disease than do younger populations [26]. According to the Center for Disease Control and Prevention (CDC) [6], in 2010 nearly $75 \%$ of US older adults have one or more chronic diseases. Also, 92\% of older adults

\footnotetext{
*Corresponding author. E-mail: youngji.chun@ttu.edu. Tel: 1-806-742-3543 (Department of Industrial Engineering).
} 
who have a chronic disease live alone, including $50 \%$ of those over 75 years old [21]. Chronic diseases require constant specialized care not only by professionals but also through self-care by the elderly themselves. Therefore, timely health information is even more critical to older populations with chronic diseases [10].

However, the elderly have more limited perceptual and cognitive capabilities, which often degrade the informative communication process compared with younger people. Working memory capacity, processing speed, and recall of information often decline with age [7]. Motor skills and visual and auditory abilities are also more limited due to aging [3]. These restrictions can interfere with the elderly's use of internet technology.

In the human factors and ergonomics fields, home healthcare has emerged as an important challenge, which includes telemonitoring, patient-physician communication, and the redesign of the telemedicine system environment for the aging [23]. While there are many studies on web information search and navigation, these topics have not been addressed in telemedicine research for the elderly [27].

The purpose of this study is to suggest future research on interface design for internet-based telemedicine systems for the elderly to aid them in making informed decisions about their health-related issues. This study also suggests developing interface design guidelines for an internet-based telemedicine system for the elderly, based on the capabilities and limitations of that population. These internet-based telemedicine systems will ensure more successful aging-in-place for the elderly and improve their overall quality of life.

\section{Methods}

First, a meta-analysis was conducted to investigate previous research on the interfaces of internet-based telemedicine systems. To find and gather existing literature, the following keywords and combinations were used: "telemedicine", "eHealth", "telecare", "homecare", "the elderly", "older adults", "interface", "usability", and "HCI". Also, a keyword "eye tracking" was searched, an area in which we were particularly interested.

Based on our analysis, we determined the types of usability studies undertaken in the field of internetbased telemedicine systems for elderly users, especially those using an eye tracking system.

\section{Analysis}

A major area of concern in HCI is the interface design within a boundary of usability. In general, usability has five important attributes: learnability, effectiveness, memorability, errors, and satisfaction $[1,5,14]$.

Usability testing allows a designer to understand and analyze user performance, and behaviors, to find potential problems of systems or products. With usability testing, sample users who represent a target user population can be observed while they are performing or interacting with a system or a product in real time [22,31].

Eye movement is an indicator of how people acquire information, attention location, and when attention is shifting from one point to another [16]. Eye movements are closely associated with cognitive processes and behavioral choices. It is possible to monitor visual information processes including accessing, searching, and processing the visual information by investigating patterns of eye movements.

An eye tracking system has been shown to be an effective method for measuring eye movements related to information processing in the domain of web-based tasks [8]. An eye tracking system provides both qualitative and quantitative data, allowing the recording of various measures such as: heat map, elapsed time to first look, fixation rate, fixation duration, number of fixation, scanpath (sequence of the fixations), area of interest (AOI), gaze \% per AOI, gaze duration, and so forth $[2,18,20]$. Based on those eye tracking measures, it is possible to acquire information about the patterns of a user's visual activities such as visual attention, search strategies, and processes while they are interacting with a web interface [9].

Many usability studies exist on various kinds of internet websites, using an eye tracking system. However, this research has mostly focused on web search engines, web library systems, web advertisements, commercial websites, and so forth. Few studies have compared age-related differences in web performances using the eye tracking system method. In particular, there exists a need for studies on internet-based telemedicine systems, using eye tracking.

There are several usability studies on the physical and cognitive capabilities of older adults and their performance on internet websites. For instance, Nielsen and his colleagues [17] conducted usability 
studies to identify the internet use characteristics of the elderly. They found that elderly users over the age of 65 have more difficulties than younger adult users when using internet-based interfaces. The elderly users showed less successful performance, more errors, more time to complete the tasks, and lower satisfaction compared with younger users. However, these studies did not specifically embrace telemedicine system websites.

Tullis [28] conducted an eye tacking study with adults over 50 years old and younger adults to identify behavioral differences in scanning web pages. The older adults significantly needed $42 \%$ more time to look at the content and $51 \%$ more time for navigation than did the younger adults. Also, the elderly users look at more areas.

On the other hand, Romano [11] found different results for performance on a website between older adults and younger adults by using an eye tracking system. Even though it was found that the younger adult group performed faster, no significant difference in accuracy of the performance or for satisfaction was found. In addition, there was no significant difference in the number of fixation between the two age groups.

There are a few studies on usability requirements for home health care systems [15]. They identified the usability problems of existing telemedicine systems and suggested a taxonomy of usability requirements. Even though these studies highlighted usability issues in telemedicine fields, none of them specifically focused on the interface design of an internet-based telemedicine system.

Telemedicine fields have focused little on usability issues of interface designs of internet-based telemedicine systems. Specifically, most current telemedicine systems were not designed for specific groups of people, but instead for a general population.

\section{Suggestions for future research}

The currently existing internet-based telemedicine systems do not consider the elderly population and are not specifically developed for the elderly user's capabilities and limitations. Therefore, examining the interfaces of current web-based telemedicine systems from an HCI perspective may provide the elderly with more effective and efficient websites for ease of use.

We suggest conducting usability testing on the interface of an internet-based telemedicine system using an eye tracking method to identify differences not only in performance level and satisfaction, but also in patterns of the visual activities between the older adults and younger adults. It would also allow us to examine which interface factors may cause those differences between different aged groups. Based on the capabilities and limitations of elderly users, design recommendations can be made for interfaces of an internet-based telemedicine system.

Based on those results, it would be possible to develop design guidelines and design standards for the interfaces of internet-based telemedicine systems for elderly users. Many older adults could then access internet-based telemedicine systems without difficulty, and better understand health-related information, so they can more effectively, efficiently, and more easily make timely and informed decisions about actions related to their health issues.

\section{References}

[1] A.D. Fisk, W.A. Rogers, N. Charness, S.J. Czaja and J. Sharit, Designing for older adults: Principles and creative human factors approaches, 2nd ed., CRC Press, Boca Raton, 2009.

[2] A. Duchowski, Eye tracking methodology: Theory and practice, 2nd ed., Springer, London, 2007.

[3] A.J. Stronge, W.A. Rogers and A.D. Fisk, Human factors considerations in implementing telemedicine systems to accommodate older adults, Journal of Telemedicine and Telecare 13(1) (2007), 1-3.

[4] B.H. Beith, Needs and requirements in health care for the older adults: Challenges and opportunities for the new millennium, in: Human Factors Interventions for the Health Care of Older Adults, W.A. Rogers and A.D. Fisk, eds., Lawrence Erlbaum Associates, Inc., Mahwah, 2001, pp. 1330

[5] B. Shneiderman and C. Plaisant, Designing the user interface: Strategies for effective human-computer interaction, 5th ed., Addison Wesley, Boston, 2009.

[6] Center for Disease Control and Prevention, Retrieved from CDC: http://www.cdc.gov/chronicdisease/stats/index.htm, 2010.

[7] D.G. Morrow and V.O. Leirer, A patient-centered approach to automated telephone health communication for older adults, in: Human Factors Interventions for the Health Care of Older Adults, W.A. Rogers and A.D. Fisk, eds., Lawrence Erlbaum Associates, Inc., Mahwah, 2001, pp. 179-202.

[8] E. Cutrell and Z. Guan, What are you looking for?: An eyetracking study of information usage in web search, in: CHI '07 Proceedings of the SIGCHI Conference on Human Factors in Computing Systems, ACM, New York, 2007, pp.407-416.

[9] G. Buscher, S. Dumais and E. Cutrell, The good, the bad, and the random: An eye-tracking study of ad quality in web search, in: SIGIR '10 Proceedings of the 33rd annual international ACM SIGIR conference on research and development in information retrieval, ACM, New York, 2010, pp. $42-49$. 
[10] J.A. Loera, Generational differences in acceptance of technology, Telemedicine and e-Health 14(10) (2008), 10871090.

[11] J.C. Romano, Using eye tracking to examine age-related differences in web site performance, Proceedings of the Human Factors and Ergonomics Society $54^{\text {th }}$ Annual Meeting, 2010, pp.1360-1364.

[12] J. Lorca, F.J. Alcazar, C.L. Sanchez, M. Romero-Cuevas, D.M. Lopez, S. Ruiz and P. Garcia-Fortea, PESCA: Open source platform for eHealth, in: eHealth: Combining Health Telematics, Telemedicine Biomedical Engineering and Bioinformatics to the Edge: CeHR Conference Proceedings 2007, B. Blobel, P. Pharow, J. Zvarova and D. Lopez, eds., IOS Press, Berlin, 2008, pp. 65-70.

[13] J.M. Machibroda, Engaging consumers in health care advocacy using the internet, in: Health communication in the new media landscape, J.C. Parker and E. Thorson, eds. Springer Publishing Company, LLC., New York, 2008, pp. 267-282.

[14] J. Nielsen and H. Loranger, Prioritizing web usability, New Riders, Berkeley, 2006.

[15] J. Singh, C. Lutteroth and B.C. Wünsche, Taxonomy of usability requirements for home telehealth systems, CHINZ '10 Proceedings of the 11th International Conference of the NZ Chapter of the ACM Special Interest Group on HumanComputer Interaction, ACM, New York, 2010, pp. 29-32.

[16] J.T-Y. Wang, Pupil dilation and eye tracking, in: A Handbook of Process Tracing Methods for Decision Research: A Critical Review and User's Guide, M. SchulteMecklenbeck, A. Kühberger and R. Ranyard, eds., Psychology Press, New York, 2011, pp. 185-204.

[17] K, Coyne and J. Nielsen, Web usability for senior citizens: 46 design guidelines based on usability studies with people age 65 and older, 2002

[18] K. Rayner and S.P. Liversedge, Visual and linguistic processing during eye fixation in reading, in: The Interface of Language, Vision, and Action: Eye Movements and the Visual World, J. M. Henderson and F. Ferreira, eds., Psychology Press, New York, 2004, pp.59-104.

[19] K.S. Rheuban, Telehealth and telemedicine technologies: Overview, benefits, and implications, in: Systems Engineering Approach to Medical Automation, R. Felder, M. Alwan and M. Zhang, eds., Artech House, Inc., Norwood, 2008, pp. 197-211.

[20] L. Lorigo, M. Haridasan, H. Brynjarsdóttir, L. Xia, T. Joachims, G. Gay, L. Granka, F. Pellacini and B. Pan, Eye tracking and online search: lessons learned and challenged ahead, Journal of the American Society for Information Science and Technology 57(7) (2008), 1041-1052.

[21] M. Alwan, Automated in-home patient monitoring: Geriatric care application, in: Systems Engineering Approach to
Medical Automation, R. Felder, M. Alwan, and M. Zhang, eds., Artech House, Inc., Norwood, 2008, pp. 213-226.

[22] M. Matera, F. Rizzo, R. Cortázar and A. Perallos, The usability dimension in the development of web applications, in: Handbook of Research on Web Information System Quality, C.C. Muñoz, M.Á. Moraga and M. Piattini, eds., Information Science Reference, IGI Global, Hershey, 2008, pp. 234-249.

[23] M.W. Scerbo, Human factors issues with automation, in: Systems Engineering Approach to Medical Automation, R. Felder, M. Alwan and M. Zhang, eds., Artech House, Inc., Norwood, 2008, pp. 5-20.

[24] P.S. Seibert, T.A. Whitmore, C.M. Patterson, C.C. Otto, P.D. Parker, N. Whitener, M.J. Ward, J. Basom and C.G. Zimmerman, Telemedicine research: Opportunities and challenges, in: Mobile Telemedicine: A Computing and Networking Perspective, Y. Xiao and H. Chen, eds., CRC Press, Boca Raton, 2008, pp. 349-366.

[25] P. Whitten, The state of telecommunication technologies to enhance older adults' access to health services, in: Human Factors Interventions for the Health Care of Older Adults, W.A. Rogers and A.D. Fisk, eds., Lawrence Erlbaum Associates, Inc., Mahwah, 2001, pp. 121-146.

[26] R.L. Glueckauf and M.L.A. Lustria, E-health self-care interventions for persons with chronic illnesses: Review and future direction, in: Health communication in the new media landscape, J.C. Parker and E. Thorson, eds., Springer Publishing Company, LLC., New York, 2008, pp. 151-242.

[27] S.J. Czaja, and C.C. Lee, The Internet and older adults Designing challenges and opportunities, in: Communication, Technology, and Aging: Opportunities and Challenges for the Future, N. Charness, D.C. Parks and B.A. Sabel, eds., Springer, New York, 2001, pp. 60-78.

[28] T.S. Tullis, Older adults and the web: lessons learned from eye-tracking, in: Universal Access in Human-Computer Interaction, Part I, HCII 2007, C. Stephanidis, ed., Springer, Berlin, 2007, pp. 1030-1039.

[29] U.S. Census Bureau, Current population survey, Annual Social and Economic Supplement 2006. www.census.gov., 2008.

[30] W. Glinkowski and M. Sawińska, Consumers use of the internet health information resources: Results of the omnimas Polish survey 2007, in: eHealth: Combining Health Telematics, Telemedicine Biomedical Engineering and Bioinformatics to the Edge: CeHR Conference Proceedings 2007, B. Blobel, P. Pharow, J. Zvarova and D. Lopez, eds., IOS Press, Berlin, 2008, pp. 251-256.

[31] W. Zhu, K-P.L. Vu and R.W. Proctor, Evaluating web usability, in: Handbook of Human Factors in Web Design, R.W. Proctor and K-P.L. Vu, eds., Lawrence Erlbaum Associates, Inc., Mahwah, 2005, pp. 321-337. 